\title{
CAPÍtulo iII \\ EL DEPORTE EN LA FORMACIÓN FÍSICA INTEGRAL \\ DEL SER HUMANO
}

\author{
Diana Paola Sáenz ${ }^{1}$ \\ Carlos Mario Toro ${ }^{2}$
}

\section{Introducción}

Este capítulo busca presentar la importancia que tiene para la formación integral el cultivo de los aspectos intelectuales y humanos del hombre: Además, presenta al cuerpo no solo como herramienta, sino como parte esencial de la comprensión del ser humano. Entender el cuerpo no es una tarea sencilla, aún más cuando este actualmente se vincula a espacios publicitarios y se ha encasillado dentro de una concepción mercantilista.

La cátedra Henri Didon busca romper con este paradigma consumista, haciendo honor a su mentor.

1 Profesional en Cultura Física, Deporte y Recreación. Universidad Santo Tomás de Bogotá.

2 Licenciado en Educación Física, Universidad de Cundinamarca. Especialista en Lúdica y Recreación para el Desarrollo Social y Cultural, Fundación Universitaria Los Libertadores. 
El cuerpo es visto como un todo, pero principalmente como una construcción social y cultural en la que, a través del deporte, se reúnen aspectos humanos y biológicos. Bajo esta perspectiva se desarrollan diferentes argumentos que cobran mayor interés para la formación física integral del ser humano en relación con el lenguaje del cuerpo. Asimismo, se plantea la controversia social sobre el futuro de los cuerpos en las nuevas concepciones acerca de su perfeccionamiento. El interés de este trabajo está en reconocer las interconexiones que existen entre la mente, el cuerpo y la formación física integral del ser humano.

\section{Naturaleza y deporte}

Pocas actividades físicas son las que realiza el individuo en un ambiente escolar universitario y laboral, menos aún en un entorno globalizado donde la tecnología realmente no propicia un ambiente de práctica deportiva en el cual el ser humano pueda interactuar.

El deporte influye en la educación, hábitos y costumbres del ser humano. Los hombres de ciencia se ocupan del cuerpo, sus movimientos, sus aptitudes y actividades, así como a la forma como el ejercicio, el juego, el entretenimiento y el descanso contribuyen a la unidad del cuerpo y de la mente.

La Universidad Santo Tomás, además de contar con una Facultad de Cultura Física, promueve el deporte universitario desde el Departamento de Bienestar Universitario, constituyéndose en un intento de reforma sustancial ${ }^{3}$. La Universidad, a través de la catedra de formación física integral Henri Didon, busca una mejor calidad de vida para sus educandos, fortaleciendo y promoviendo el ejercicio físico como un hábito de vida saludable desde tres áreas fundamentales: condición física, salud y psicología, para que sus experiencias vividas se conviertan en factores importantes en el aprendizaje y formación integral y de este modo se vea reflejado en su vida profesional y social ${ }^{4}$. La formación humanista dentro del plan de estudios es transversal, en coherencia y alineación con la filosofía y los principios de la misión institucional.

3 Mike Forero-Nougués, Historia de tres mundos: cuerpo, cultura y movimiento. Reflexiones de cultura física (Bogotá: Universidad Santo Tomás, 2004), 59.

4 Departamento de Humanidades y Formación Integral, Proyecto de cátedra formación física integral Henri Didon, (Bogotá: Universidad Santo Tomás, 2015). 


\section{Fray Henri Didon y el deporte}

Henri Didon fue un sacerdote dominico francés (1840-1900), reconocido como predicador, pedagogo y pionero del movimiento deportivo internacional y de los Juegos Olímpicos Modernos, así como creador del lema "citius, Altius, fortius" (más rápido, más alto, más fuerte) ${ }^{5}$, el cual fue establecido oficialmente como lema olímpico en el Congreso de París en 1994.

Estableció el deporte como una de las prácticas educativas principales de la escuela. Compartió mesa con los principales líderes deportistas de la época, como Georges de Saint-Clair, o Pierre de Coubertin en el marco del Congreso Olímpico Internacional (1897) justamente Coubertin es llamado "padre de los Juegos Olímpicos Modernos".

Es por ello que la cátedra de cultura física de la Universidad Santo Tomás ahora recibe el nombre de Formación física integral Henri Didon, en su honor.

\section{Deporte y modernismo}

Las nuevas generaciones pasan la mayor parte de su tiempo en reposo y se calcula que durante 150 mil horas de su vida o más, los jóvenes permanecen estáticos frente a la televisión o a las computadoras, en quietud sin inversión de energía. Contrarrestar esta situación, mediante el movimiento del hombre, es no solamente una preocupación social del momento sino una necesidad inaplazable .

Dentro de lo que se denomina modernismo o progreso de la tecnología, el individuo se encuentra en un estado de comodidad, bajo el estímulo de la pereza, inclinándose por la quietud y confundiéndola con el confort. Es por ello que el deporte se ha generalizado, convirtiéndose en una necesidad para el hombre, con el fin de cultivar una adecuada condición física que le permita al cuerpo desenvolverse con mayor propiedad. Es parte primordial del desarrollo integral y de la lucha contra el inmovilismo.

5 Fray Henri Didon, Influence morale des Sports athlétiques. Discours prononcé au Congrès Lympique du Havrele 29 juillet 1897, (Proyecto Gutenberg, 2004), http://goo.gl/xBPtOf

6 Mario Ramírez Alfonso et al, El Barón Pierre de Coubertin, padre de los Juegos Olímpicos de la era moderna, Revista Digital Vol.18, No 187 (2013), http://goo.gl/uV7GZY.

7 Forero-Nougués, Op. Cit., 113. 
La cultura física (cuerpo, cultura y movimiento) puede ser una manifestación moderna, posmoderna y antigua. El ejercicio es la actividad más antigua del hombre después de la alimentación. El deporte y la actividad física a partir del siglo XIX han sido vistos como uno de los fenómenos sociales de mayor impacto y más necesarios para el hombre.

\section{Deporte y humanismo}

Humanismo significa confiar en el hombre, tener fe en el hombre y comprometerse para que su vida sea digna y feliz, justa y dichosa. Aunque haya habido a lo largo de la historia diversas modalidades de humanismo, todas ellas han pretendido el esclarecimiento y la realización plena de la realidad humana, de lo que han entendido como verdadera vida humana, enfrentándose con los obstáculos y amenazas que en cada momento impedían una vida buena en concordancia con el ser humano ${ }^{8}$.

"Una educación realmente humanista no puede nunca despreciar el deporte (...) El humanismo ve en el deporte un medio de aprender a dominar y a organizar los movimientos. Es, en último término, el triunfo de la razón sobre los miembros y la manifestación concreta de la armonía en los movimientos corporales (...) El deporte también es una escuela de sociabilidad y cooperación"

La problemática actual de la formación humanística esta suscitada por el impacto de la ciencia y la tecnología, reforzada muchas veces por la mentalidad tecnocrática, el tecnicismo y una fuerte mentalidad pragmática orientada solo hacia la actividad instrumental. De este modo surge el dualismo educativo: la cultura científica y la cultura humanística.

El humanismo, cualquiera que sea su calificación, es ante todo creer en el hombre y defenderlo sobre todas las cosas, bajo la firme convicción de que se trata de una realidad que "no vale para nada" y, por consiguiente es la más valiosa y preferible por su dignidad. De ahí que todo lo demás debe ponerse a su servicio, pues de lo contrario el hombre queda instrumentalizado y degradado ${ }^{10}$.

8 Eudoro Rodríguez Albarracín, ¿Qué es el humanismo? Problemática de la formación humanística, Análisis: revista colombiana de humanidades $\mathrm{N}^{\circ} 72$ (2008): 103.

9 José Guadalupe de la Mora Ledesma, Esencia de la filosofía de la educación (México: Editorial Progreso, 2004), 236.

10 Ibíd., 237. 
Entre las diversas ciencias que se relacionan con la práctica del deporte encontramos la antropología, la cual nos conduce a examinar toda la historia del hombre desde el punto de vista de su anatomía y fisiología, la genética, la biología y la conducta de los humanos. Otra ciencia con la que la práctica deportiva tiene relación directa es la economía. El deporte no solo tiene una faz de entretenimiento sino que aparece como una actividad económica de gran magnitud a escala global. Adam Smith, padre de la economía, sostuvo que el hombre, su cuerpo, su movimiento y su talento se convierten en el centro de la economía política.

La cultura física, en todas sus ramas es en la actualidad un proyecto económico. La industrialización deportiva es una fuente de acumulación de grandes capitales que por las mismas fuerzas de la economía, requiere una alta especialización. Las actividades físicas como los juegos, los deportes y la recreación se han convertido en una grande y poderosa industria tanto en países industrializados como los que se encuentran en vía de desarrollo ${ }^{11}$.

\section{El cuerpo}

En palabras de Braidoti, el cuerpo o la corporización del sujeto no debe entenderse ni como una categoría biológica ni como una categoría sociológica, sino más bien como un punto de superposición entre lo físico, lo simbólico y lo sociológico ${ }^{12}$.

La preocupación por el propio cuerpo y por las muchas situaciones de los cuerpos, dadas circunstancias como: los ciclos vitales, la vejez, las demandas estéticas, los cuidados de la salud y la enfermedad conducen a los individuos, según cada circunstancia y cada interés, a realizar prácticas sobre el cuerpo.

Podemos concluir, afirmando que el cuerpo ha tenido una evolución a lo largo de la historia, que la educación física es sinónimo de educar nuestro cuerpo, de ayudarnos a comprender y desarrollar el potencial físico e intelectual como afirma Freile: desarrollarnos e interactuar dentro de la comunidad social.

11 Forero-Nougués, Op. Cit., 118.

12 Rosi Braidotti, Sujetos nómades. Corporización y diferencia sexual en la teoría feminista contemporánea, (Buenos Aires, Paidós, 2000), 29. 
Jean Baudrillard ${ }^{13}$ considera que la lógica social del consumo es una lógica de consumo de signos, donde el cuerpo aparece dentro del abanico de los objetos de consumo, y bajo el signo de la liberación sexual, el cuerpo comienza a ser objeto de numerosas inversiones narcisistas, físicas y eróticas. Pero lo más importante en todo este proceso es que el cuerpo parece haber sustituido al alma como objeto de salvación. La propaganda y la publicidad se encargan continuamente de recordarnos que tenemos un solo cuerpo y que hay que salvarlo y cuidarlo. Lo que Baudrillard ha querido demostrar en su análisis es que las estructuras actuales de producción y consumo proporcionan al individuo una doble representación de su cuerpo: como una forma de capital y como fetiche, es decir, el cuerpo moderno se exhibe como una forma de inversión y signo social a la vez ${ }^{14}$.

El cuerpo se ha convertido en parte de un proyecto en el que hemos de trabajar, proyecto que va vinculado a la identidad del yo de una persona. El cuidado del cuerpo no hace referencia solo a la salud, sino también a sentirse bien; nuestra felicidad y realización personal, cada vez más, están sujetas al grado en que nuestros cuerpos se ajustan a las normas contemporáneas de salud y belleza.

El cuerpo "ideal” es mucho más importante para la mujer que para el hombre. Por ello, las mujeres obesas son más discriminadas socialmente y presionadas para hacer más dieta que los varones obesos, y son ellas las que llenan las clínicas de control de peso ${ }^{15}$.

Featherstone distingue dos tipos de exigencias con respecto al cuerpo: que el cuerpo interior funcione bien (esté sano y en forma) y que la apariencia sea cuidada. Así, el cuerpo es un signo, es un mensaje que habla de su propietario ${ }^{16}$.

\section{Evolución del cuerpo}

Los griegos empezaron a racionalizar el pensamiento y de esta manera comenzó el dualismo cuerpo-alma, llegando a la individualización. Con esta separación, el hombre comienza a

13 Jean Baudrillard en Martínez Barreiro (2004): 131-132.

14 Ana Martínez Barreiro, La construcción social del cuerpo en las sociedades contemporáneas, Papers: revista de sociología $\mathrm{N}^{\circ} 73$ (2004): 131.

15 Ibíd., 140.

16 Featherstone en Martínez Barreiro (2004): 139-142. 
separarse de su cuerpo y de sus mitos, cuestionando de dónde provienen las sensaciones, para así dudar y tratar de reaccionar al mundo.

Con el advenimiento del siglo $\mathrm{V}$ a. C., el cuerpo se incorpora en el ideal educativo griego para los ciudadanos; educación que buscaba el equilibrio integral para todas las artes, la gimnasia, la ciencia, la música, la filosofía, la política, las leyes y estaba orientada a formar un ciudadano virtuoso. El cuerpo era concebido como una carga, como un sufrimiento, sometido a la disciplina del orden establecido por la iglesia, supeditado a la salvación del alma, sumiso, compasivo, abstemio de dolor y de vida.

A partir de la Revolución Francesa, se promovieron nuevos valores, la modernidad se instala en las principales ciudades de Europa, exponiendo al individualismo urbano y la revolución industrial como principal característica. El cuerpo se alineaba con los mecanismos de producción y se lo veía como una máquina para el trabajo.

Por otro lado, la Revolución Industrial en Inglaterra dio como resultado el nacimiento de otra escala social: la burguesía industrial. A esta burguesía industrial, junto con la aristocracia que gobernaba el país, le sobraba mucho tiempo libre y lo aprovechaba para ejercitar su cuerpo, gestando el nacimiento del deporte moderno. Las pruebas atléticas (carrera de fondo y saltos y lanzamientos) y el golf ya eran reconocidos con el nombre de deportes y eran profesionales.

Este cambio de concepción del cuerpo produjo una valoración más importante, llena de expresiones subjetivas, realzando el cuerpo colectivo e individual y nacen así los Juegos Olímpicos Modernos ${ }^{17}$.

En los años setenta perduraba una idea del cuerpo ligada al ejercicio de lo sensorial y la actividad física que se expresaba en la vida cotidiana. Muchos concurrían al trabajo por sus propios medios (caminando) o con instrumentos sencillos de locomoción (bicicleta) tratando de cumplir siempre el mismo camino, habitando el trayecto. El hombre aún tenía anclaje en su cuerpo y la comunidad.

La década de los ochenta marcaría el inicio de la explotación del cuerpo, algo que llega hasta nuestros días. El cuerpo es sometido al máximo con distintos tipos de entrenamiento para cualquier experimentación científica, acrobacia y/o técnica motriz. Todo esto fomentado por los avances tecnológicos, por la expansión de los medios de comunicación que invaden todos los campos sociales, como la danza, la gimnasia, la familia y el deporte. Se produce una reelaboración

17 Germán Alejandro Ruggio, El cuerpo: la afirmación a lo largo de la historia como formador de la identidad, Revista Digital Vol. 16 No 160 (2011), http://goo.gl/c9JKnh 
de la definición de intimidad, fomentada por el creciente individualismo. Esta visión está ligada al endiosamiento de la apariencia física como forma de éxito.

Con la llegada de los ańos noventa, los avances de la modernidad no eran los esperados y empezaban a dejar consecuencias, la posmodernidad y la velocidad vivenciada asumen un valor creciente. En los hogares se advierte un hombre cómodo, sentado o acostado frente a la pantalla del televisor o de la computadora, remplazando las ventanas o las puertas, aprovechando la comodidad de los controles remotos o teléfonos móviles, convirtiéndose la tecnología en una parte más de nuestros cuerpos.

Este nuevo siglo propone una diferencia socioeconómica con respecto a los cuidados del cuerpo. Las clases se acomodan y tienen acceso a tiempo libre para descansar en lugares propios y sociales repletos de comodidades corporales: gimnasios, máquinas de deportes extremos, tratamientos de masajes y de belleza, saunas y jacuzzis. Todo esto promueve una mejor calidad de vida, los cuidados profesionales en clínicas privadas de salud para tratar de salvar el cuerpo de la vejez, de la muerte diaria.

Es así, como Forero-Nougués plantea que mientras que, en las clases menos acomodadas el cuerpo es vivido como una herramienta de trabajo, de subsistencia, resignados a los planes sociales de salud y bienestar de los estados provinciales y nacionales, diferente a la clase acomodada, sin descanso más que el que brinda el sueño, exponiendo la vida en salas de emergencia repletas de cuerpos, virus y violencia. El cuerpo en las clases desfavorables es vivido con violencia, con furia y se exterioriza en el abandono y la desazón, la desilusión por el futuro y en el día a día.

Muchos nińos latinoamericanos se ven precisados a trabajar para ayudar al sostenimiento de sus familias y tras esfuerzos estimulados por la necesidad, algunos ensayan la posibilidad de dar el salto al deporte profesional para ubicarse, repentinamente, en un alto nivel económico que supera el ingreso medio que puedan obtener algunos de los profesionales de carreras distintas al deporte.

En las grandes ciudades de Colombia, a donde llegan centenares de familias desplazadas por la violencia, se ha observado un fenómeno muy particular: los nińos practican ejercicios de acrobacia en las calles, haciendo toda clase de piruetas frente a los vehículos que se detienen en los semáforos. Se ganan la vida mediante el "espectáculo deportivo"18.

18 Forero-Nougués, Op. Cit., 96. 


\section{Cuerpo y mente}

La fortaleza mental es uno de los requisitos más importantes y necesarios en toda actividad deportiva. Sin esta, difícilmente podremos hablar de un deportista exitoso a pesar de una buena técnica y preparación física y más bien se hablará de un deportista promedio o de repente bueno, pero que no llegará más allá. Este punto es definitivamente uno de los fundamentales a trabajar en la preparación psicológica de todo deportista en general, pero sobre todo en los competitivos y aún más, los de élite. A su vez, es una de los más difíciles de lograr.

La fortaleza mental es uno de los requisitos más importantes y necesarios en toda actividad deportiva. Un deportista debe educar su mente para que lo alimente de los pensamientos adecuados. El atleta necesita pensamientos positivos. El cuerpo hace lo que la mente le dice. Si esta le dice "no puedes hacerlo", el deportista no lo va a hacer, porque su mente le dice a su cuerpo que no es posible lograrlo, pero si le dice: "tú puedes hacerlo", se incrementará el rendimiento, alcanzando muchas veces mayor importancia y relevancia que el aspecto técnico. El deportista juega como piensa, eso es lo que permitirá rendimientos picos. El atleta que piense mal definitivamente no obtendrá los resultados deseados, en cambio al pensar bien podrá rendir de una manera mucho mejor ${ }^{19}$.

\section{Cuerpo y corporeidad}

La corporeidad puede asociarse a la motricidad, ya que el ser humano experimenta con su cuerpo de manera práctica. La corporeidad y la motricidad son inseparables, no existen una sin la otra. Si bien en el análisis científico son elementos con definición propia, en la realidad práctica el cuerpo no puede concebirse sin vida, sin movimiento.

La motricidad está presente en la cotidianidad del hombre, sus acciones, sentimientos y finalidad, interactuando y expandiéndose en el tiempo, en busca de la trascendencia de sus actos con el medio social y natural. La motricidad da sentido a la corporeidad y viceversa.

El movimiento siempre ha servido como método para que las distintas sociedades moldeen el cuerpo humano, está presente en los currículos escolares, en las ideologías de cada momento

19 Dante Nieri Romero, La importancia de la fortaleza mental en los deportes, Revista Digital Vol. $10 \mathrm{~N}^{\circ} 90$ (2005), http://goo.gl/iB3hYN 
histórico, reduciendo al hombre a disciplina o descubrimiento, en busca de un control social absoluto.

Se puede definir al hombre desde las siguientes dimensiones:

- La corporeidad: desde y a partir del cuerpo me proyecto al mundo.

- La motricidad: interioriza y humaniza el movimiento.

- La comunicación y cooperación: afirma el nosotros, nos conecta, nos une al estar en el mundo.

- La historicidad: vivir nuestro presente, proyectándonos al futuro esperanzador sin olvidar de dónde venimos ni nuestro pasado.

- La libertad: entender nuestras necesidades para convertirnos en seres reflexivos, históricos y autónomos del propio medio.

- La noosfera: buscar la totalidad humana por medio de los saberes espirituales y culturales.

- La trascendencia: buscar hacer para crecer y ser mucho más.

A partir de estas dimensiones el cuerpo no se reduce a una instancia biológica, sino a un ser histórico-social, biológicamente activo ${ }^{20}$.

La corporeidad, representa el modo de ser del hombre bajo circunstancias históricas, siendo la educación física su herramienta más importante.

Separando el concepto de educación física, encontramos históricamente que la palabra educación estará asociada a distintos términos y sucede lo mismo con la palabra física. Es decir, el concepto de educación física encierra una connotación en el imaginario social y cultural que se ve lleno de poder y según las concepciones ideológicas propias del concepto de cada estado o nación.

\section{Cuerpo y corporalidad}

La mayoría de los estudios definen nuestro cuerpo como algo objetivo, concreto, que se puede medir con límites precisos; sin embargo, lo que llamamos esquema corporal es la idea que tenemos del mismo, por lo tanto es algo subjetivo y sujeto a posibles modificaciones.

La corporalidad se constituye en un instrumento de expresión de nuestra propia personalidad, también sirve para tomar contacto con el exterior, comparándonos con otros cuerpos y objetos,

20 Ruggio, Op. Cit. 
por lo que se puede hablar, dentro del esquema corporal, del cuerpo objeto, es decir, de la representación aislada que nos hacemos de nuestro cuerpo en sí mismo, y del cuerpo vivido, que se refiere a la forma en que nuestra corporalidad se manifiesta en nuestras relaciones humanas y en la socialización.

Nuestros cuerpos no son solo el lugar desde el cual llegamos a experimentar el mundo, sino que a través de ellos llegamos a ser vistos en él ${ }^{21}$. Para Merleau Ponty el yo está ubicado en el cuerpo, que a su vez está ubicado en el tiempo y en el espacio. A veces somos conscientes de nuestros cuerpos como objetos que se han de mirar en espacios sociales concretos, mientras que en otros, como el hogar, no consideramos nuestros cuerpos como objetos que han de ser contemplados. En los espacios públicos podemos sentir que estamos en primer plano, mientras que, cuando estamos en casa, nos encontramos entre bastidores.

La vestimenta es siempre contextual, es decir, se adapta a situaciones muy distintas. Puede suceder que haya algunos momentos en los que el acto de vestirse constituya un acto irreflexivo, similar a ir de compras o a recoger a los niños al colegio,mientras que en otros, el acto de vestirse es consciente y reflexivo, como vestirse para una entrevista de trabajo o una reunión importante. El tiempo y el espacio ordenan nuestro sentido del yo en el mundo, nuestras relaciones y encuentros con los demás y, también, la forma de cuidar de nuestros cuerpos.

En Occidente, la práctica cotidiana de vestirse implica ser consciente del tiempo, porque la moda ordena la experiencia del yo y del cuerpo en el tiempo, y esta ordenación del tiempo se da especialmente a través del sistema de la moda que se organiza en dos temporadas: primaveraverano y otońo-invierno. Las revistas de moda, a su vez, congelan el flujo de las prácticas cotidianas del vestir y lo ordenan en distintas categorías: pasado, presente y futuro.

\section{Cuerpo, ciencia y tecnología}

El progreso científico y médico genera soluciones técnicas que cambian el significado del cuerpo. Estas técnicas han permitido que tras la muerte de una persona se puedan donar sus órganos y seguir viviendo en otro cuerpo o pueden dejar una parte de su material genético en espera de que algún día una mujer logre una gestación.

21 Martínez Barreiro, Op. Cit., 135. 
Nacer y morir son experiencias que hasta hace poco tiempo marcaban los límites del tránsito terrenal de todo ser humano. El nacimiento suponía el inicio de una identidad social de un ser humano y la muerte era el fin. En el momento en que el corazón se paraba, se consideraba que todos los órganos vitales habían dejado de funcionar y la persona pasaba a ser un cadáver, un cuerpo inerte, sin vida. La definición del principio y del fin del cuerpo humano determinada por las leyes biológicas ha dejado de ser una condición inamovible debido al paulatino avance de la medicina.

Gracias a las nuevas tecnologías médicas, el alcance de los expertos raya en lo que hace pocos años se consideraba ciencia ficción. Al poder modificar las condiciones que definen el inicio o el fin del cuerpo se crean unas posibilidades en el ámbito de algunas enfermedades mortales y de la reproducción, pero lo más significativo es que se genera la necesidad de redefinir la relación entre el cuerpo humano y la identidad individual. ${ }^{22}$

\section{Ídolos deportivos y su formación integral}

El deporte en su máxima expresión crea ídolos, paradigmas, personas ejemplares que logran que el público se contagie del entusiasmo natural de las grandes pasiones.

¿Cómo sobrepasar nuestros propios límites? La cabeza suele ser más perezosa que el resto del cuerpo. Así como los monjes se alejan del cuerpo para encontrar una verdad, existen casos de deportistas que son similares, pero a la inversa, es decir, personas que se alejan de la mente y se refugian en el cuerpo para lograr una verdad del cuerpo en movimiento; piensan en el cuerpo que libra batallas brutales y lo trabaja como territorio de pensamiento.

Es otra forma de ver el tiempo, ellos definen vidas en un par de horas; encontramos, por ejemplo a Damian Blaum, nadador quien en 8 horas y 17 minutos nadó 88 kilómetros en el maratón acuático internacional Hernandarias Paraná y se llevó la marca más rápida de la historia. "El dolor pasa y después viene la gloria,... Llegué, gané, lo hice, siento un calor que me sube desde el estómago y vomito con fuerza”23.

22 Nieri Romero, Op. Cit.

23 Federico Bianchini, Desafiar al cuerpo: del dolor a la gloria. El deporte llevado al extremo, (Buenos Aires: Aguilar, 2014), 11. 
María Inés Matto, que nadaba en aguas heladas, dice que en el deporte no se analiza cómo ganar, perder, esforzarse más, rendirse o seguir, pero detrás de ello se esconde algo mucho más mental. El que va a correr una maratón de 90 kilómetros pasa meses pensando en lo que come, cuánto duerme, si sale en la noche o no. Posteriormente, en la carrera el cuerpo deja de responder y tienen que hacer algo para no abandonar, que sería lo más racional. Entonces sacan la cabeza del cuerpo y se evaden para que el cuerpo fluya y el dolor se vaya ${ }^{24}$.

Ellos se engañan a sí mismos para someterse a presiones tan duras. Por ejemplo, Gisella Dulko, decía: "Yo a los 30 dejo el tenis, no me importa en qué momento de mi carrera esté". Saben que en un punto es mentira, pero necesitan pensar que en algún momento viene la tranquilidad, que no es en ese momento, pero que viene. Otros deportistas dicen que eliminan una angustia a través del deporte y también está la oleada de endorfinas que viene después, que es un placer que se va volviendo necesario. Les pasa a los deportistas de élite: llega un punto en que el cuerpo no da más, pero ellos lo siguen necesitando y consumen drogas para alcanzar ese placer.

La historia de Cristian Gorbea, gerente del Banco Hipotecario, que corriendo una carrera en el Cerro Champaquí, Córdoba, se cae en una cornisa de un metro por tres metros y queda atrapado 42 horas.

Él sabía que la única forma que tenía de sobrevivir era a través de la paciencia, porque si intentaba escalar, seguramente iba a terminar desbarrancando, debía soportar estar solo, en el medio de la nada mientras era rescatado.

Un nadador compitiendo (8 horas y 17 minutos) donde vomita y hace sus necesidades, “¿por qué?” Él responde que se sufre mucho. “¿Qué sucede con el dolor?”. Él aduce “Trato de sacar la cabeza del río, me imagino otra situación, algún recuerdo de mi infancia, comiendo un asado con mi familia o una fiesta de cumpleaños. De esa forma me olvido del dolor, del sufrimiento y del cuerpo"25.

Es sorprendente el entrenamiento mental de los deportistas. En el yatching, por ejemplo, entrenan sentados en una alfombra, deben imaginar la situación del día de la competencia: el río, el viento, las olas. Los deportistas empiezan a moverse y transpirar porque el deporte es automatismo. Se trata de prepararse para todos los problemas, previsualizar todos los detalles de la escena.

La confianza en sí mismo es vital para cualquier deportista, un deportista con una pobre confianza nunca podrá rendir su verdadero potencial. Un elemento muy utilizado para alcanzar

24 Ibíd.

25 Ibíd., 35. 
esto antes de las competencias son los llamados rituales o "cábalas", la creencia de que un hechoacción incrementa el rendimiento del atleta es real porque este lo cree así.

El montañista Aron Ralston — quien pudo salvarse después de que una piedra suelta golpeara su brazo derecho cuando una roca le cayó encima mientras practicaba montañismo en el Blue John Canyon en Utah-, a lo largo de su suplicio, Ralston recordó amigos, familia y a las dos excursionistas que conoció (Amber Tamblyn y Kate Mara) antes de su accidente.

A lo largo de los siguientes cinco días, Ralston batalla con los elementos y sus propios demonios, hasta que finalmente descubre que tiene el valor y la entereza para zafarse por cualquier medio que sea posible, bajar por una ladera de 20 metros de alto y caminar por más de doce kilómetros hasta que es finalmente rescatado. Sin embargo, este aventurero considera que a pesar de haber dejado una parte de su cuerpo en ese lugar, nunca perdió nada y aunque el accidente cambió para siempre su vida y el dolor que sintió fue indescriptible, salió de su "tumba" y regresó a la vida.

"Yo debí cortarme el brazo y puede que suene bastante horrible, sin embargo pienso que fue algo hermoso, lo considero como una bendición (...)"26.

La mayoría de las diferentes culturas, grupos y personas consideran la vida humana algo sagrado, pero el valor de la vida humana y su centralidad se interpretan de diferente manera. De ahí que la eutanasia plantee verdaderos dilemas morales entre el valor primordial de la vida humana y el respeto por la autonomía de la persona y evitar el sufrimiento. La eutanasia toma la forma de asistencia al suicidio o de suspensión de los tratamientos médicos ofrecen soporte vital en la idea de que es preferible la muerte a continuar una existencia de sufrimiento insoportable o a mantener una vida carente de aprecio por sus condiciones de deterioro. Las decisiones sobre la vida y la muerte son las más cruciales, tanto por su extraordinaria relevancia moral como por su complejidad.

La argumentación jurídica actual considera que el cuerpo es para el derecho una cosa, optando por la opción más clásica del dualismo, reconociendo que no es el cuerpo lo que le interesa para definir la personalidad, sino la razón y la voluntad.

26 Theo González Castaño, No perdi un brazo, volvi a la vida: Aron Ralston (Bogotá: El Espectador, 2014), http://goo.gl/kAeFL1. 


\section{Conclusión}

En la actualidad, el cuerpo está sujeto a fuerzas sociales, económicas, éticas, morales, religiosas entre otras, las cuales se experimentan en las comunidades tradicionales. Por una parte, los discursos contemporáneos sobre salud y la imagen vinculan el cuerpo y la identidad, y sirven para promover ciertas prácticas de cuidados corporales típicas de la sociedad contemporánea. Por otra parte, el cuerpo se ve fragmentado por los avances de la ciencia y de la tecnología. No obstante, a través de estos avances, el hombre puede cortar el lazo que conecta la vida de la especie con el de la reproducción como proceso natural. La ciencia enfrenta a la sociedad con un conjunto de dilemas (aborto, eutanasia, clonación) difíciles de resolver.

Si el propósito de las humanidades es mediar y asumir los procesos de la humanización y la deshumanización, se justifican en el plano educativo en la medida que conduzcan a una formación integral de la persona dentro de una concepción integral de la educación.

Se deben formar y preparar profesionales con clara conciencia histórica, social, deportiva, con influencia ética y humanista que los motive al compromiso del cambio con una visión determinada del hombre y la realidad.

\section{Referencias}

Bianchini, Federico. Desafiar al cuerpo: del dolor a la gloria. El deporte llevado al extremo. Buenos Aires: Aguilar, 2014.

Braidotti, Rosi. Sujetos nómades. Corporización y diferencia sexual en la teoría feminista contemporánea. Buenos Aires, Paidós, 2000.

Departamento de Humanidades y Formación Integral. Proyecto de cátedra formación física integral Henri Didon. Bogotá: Universidad Santo Tomás, 2015.

Didon, Henri. Influence morale des Sports athlétiques. Discours prononcé au Congrès Lympique du Havrele 29 juillet 1897. (Proyecto Gutenberg, 2004). http://goo.gl/xBPtOf

Forero-Nougués, Mike. Historia de tres mundos: cuerpo, cultura y movimiento. Reflexiones de cultura física. Bogotá: Universidad Santo Tomás, 2004.

González Castaño, Theo. No perdí un brazo, volvi a la vida: Aron Ralston. Bogotá: El Espectador, 2014. http://goo.gl/kAeFL1 
Martínez Barreiro, Ana. La construcción social del cuerpo en las sociedades contemporáneas. Papers: revista de sociología $\mathrm{N}^{\circ} 73$ (2004): 127-152.

Mora Ledesma, José Guadalupe de la. Esencia de la filosofía de la educación. México: Editorial Progreso, 2004.

Nieri Romero, Dante. La importancia de la fortaleza mental en los deportes. Revista Digital. Vol. $10 \mathrm{~N}^{\circ} 90$, 2005. http://goo.gl/iB3hYN

Ramírez Alfonso, Mario, Gustavo Adolfo Oliveros Soriano, Fausto Cabrera Martínez, Jondrette Díaz Socorro, y Carlos Baños Prieto. El Barón Pierre de Coubertin, padre de los Juegos Olímpicos de la era moderna. Revista Digital. Vol.18, No 187, 2013. http://goo.gl/uV7GZY

Rodríguez Albarracín, Eudoro. ¿QQué es el humanismo? Problemática de la formación humanística. Análisis: revista colombiana de humanidades $\mathrm{N}^{\circ} 72$ (2008): 89- 104.

Ruggio, Germán Alejandro. El cuerpo: la afirmación a lo largo de la historia como formador de la identidad. Revista Digital Vol. 16 No 160, 2011. http://goo.gl/c9JKnh 